\title{
A study of tube-like surfaces according to type 2 Bishop frame in Euclidean space
}

\author{
Hossam S. Abdel-Aziz
}

\begin{abstract}
The main goal of this paper is the study of the classical differential geometry of a special kind of tube surfaces, so-called tube-like surface in 3dimensional Euclidean space $\mathbf{E}^{3}$. It is generated by sweeping a space curve along another central space curve. In particular, the type 2 Bishop frame is considered and some important theorems are obtained for that one. Finally, an application is presented and plotted using computer aided geometric design.
\end{abstract}

Mathematics Subject Classification (2010): 53A04, 56B34, 53B25.

Keywords: Tube-like surface, Weingarten and linear Weingarten surfaces, Gaussian and mean curvature functions, second Gaussian curvature, Type 2 Bishop frame, Euclidean space.

\section{Introduction}

In the study of the differential geometry of submanifolds, it is prevalent to examine different types of curvature conditions. Accurately, one is excited to determine all submanifolds satisfying such a condition. A delectable curvature property to study for a surface $\Sigma: \phi=\phi(u, v)$ in an Euclidean space $\mathbf{E}^{3}$ which requires the existence a functional relationship $\Gamma\left(k_{1}, k_{2}\right)=0$ between the principal curvatures is called Weingarten surfaces or $W$-surfaces. With the use of the Gaussian and mean curvatures $K$ and $H$ respectively, we can redefine $W$-surfaces, as surfaces satisfying $\Gamma(K, H)=0$, or, equivalently, the corresponding Jacobian determinant is identically zero, i.e.,

$$
\Gamma(K, H)=\left|\frac{\partial(K, H)}{\partial(u, v)}\right|=0 .
$$

Besides, if $\phi$ satisfies a linear equation $a K+b H=c, a, b, c \in \mathbb{R},(a, b, c) \neq(0,0,0)$, then it said to be a linear Weingarten surface or $L W$-surface.

Here, when $a=0$, a $L W$-surface $\phi$ becomes a surface with constant mean curvature. Also, when $b=0$, a $L W$-surface $\phi$ will be a surface with constant Gaussian curvature. From this point, the linear Weingarten surfaces represent a natural 
generalization of surfaces which have constant mean curvature or constant Gaussian curvature.

As well known, in the differential geometry of curves, the curves are investigated by the well know Frenet-Serret equations because they are considered as the path of a moving particle in the Euclidean space. On the other hand, some researchers aimed to determine another moving frame for a regular curve. In 1975, Bishop pioneered "Bishop frame" by means of parallel vector fields. This frame is also called a "parallel" or "alternative" frame of the curves [4]. The important application of Bishop frame is that it is used in the area of biology and computer graphics. For example, it may be possible to compute information about the shape of sequences of DNA using a curve defined by Bishop frame. Also, it may provide a new way to control virtual cameras in computer animations [20]. In the present time a good deal of research has been done using Bishop frames $[5,6,7,10,25]$. Because of the importance of this frame, the authors in [29] introduced a new version of the Bishop frame and called it a type 2 Bishop frame which was studied in $[11,19]$.

Beside the above some geometries were interested with the study of Weingarten surfaces. For example in [27, 28], the Weingarten surfaces in Euclidean space were introduced by J. Weingarten in the context of the problem of finding all surfaces isometric to a given surface of revolution. Further, applications of these surfaces on computer aided design and shape investigation can be presented in [26]. Also, in the three dimensional Euclidean space, Munteanu and Nistor [17] and Lopez [14, 15] studied polynomial translation and cyclic linear Weingarten surfaces, respectively. In addition, Ro and Yoon [21] studied a tube of Weingarten types satisfying some equation in terms of the Gaussian curvature, mean curvature and second Gaussian curvature. Kim and Yoon [13] classified quadric surfaces in Euclidean 3-space in terms of the Gaussian curvature and the mean curvature while Yoon and Jun [31] classified non-degenerate quadric surfaces in Euclidean 3-space in terms of the isometric immersion and the Gauss map. Recently, in [23], the author was studied Weingarten tube-like surfaces in Euclidean 3-space. In a Minkowski 3-space $\mathbf{E}_{1}^{3}$, a classification of these surfaces is given in $[1,2,8,12,16,24]$.

This paper is devoted to use the new version of type 2 Bishop frame which was given in [29] to introduce a study for parametrization of a tube-like surface satisfying the Jacobi condition in Euclidean 3-space $\mathbf{E}^{3}$. Moreover, for $A, Q \in\left\{K, H, K_{I I}\right\}$, we discuss the $(A, Q)$-Weingarten and linear Weingarten for that one. Thus, the geometry of such surface in terms of its intrinsic geometric formulas is established. An application of this surface is considered and plotted.

\section{Geometric preliminaries}

Let $\mathbf{E}^{3}$ be a Euclidean 3 -space with the scalar product given by

$$
g=d x_{1}^{2}+d x_{2}^{2}+d x_{3}^{2},
$$

where $\left(x_{1}, x_{2}, x_{3}\right)$ is a standard rectangular coordinate system of $\mathbf{E}^{3}$. In particular, the norm of a vector $U \in \mathbf{E}^{3}$ is given by $\|U\|=\sqrt{\langle u, u\rangle}$. If $u=\left(u_{1}, u_{2}, u_{3}\right)$ and 
$v=\left(v_{1}, v_{2}, v_{3}\right)$ are arbitrary vectors in $\mathbf{E}^{3}$, we define the vector product of $u$ and $v$ as the following

$$
u \wedge v=\left(u_{2} v_{3}-u_{3} v_{2}, u_{3} v_{1}-u_{1} v_{3}, u_{1} v_{2}-u_{2} v_{1}\right) .
$$

Let $\alpha=\alpha(t): I \rightarrow \mathbf{E}^{3}$ be a space curve in $\mathbf{E}^{3}$. Denote by $\{e(t), p(t), q(t)\}$ the moving Frenet frame along the curve $\alpha$, then the Frenet formulas are given by [22]

$$
\frac{\partial}{\partial t}\left[\begin{array}{c}
e(t) \\
p(t) \\
q(t)
\end{array}\right]=\left[\begin{array}{ccc}
0 & \kappa(t) & 0 \\
-\kappa(t) & 0 & \tau(t) \\
0 & -\tau(t) & 0
\end{array}\right]\left[\begin{array}{c}
e(t) \\
p(t) \\
q(t)
\end{array}\right],
$$

where $e=\frac{\alpha^{\prime}(t)}{\left\|\alpha^{\prime}(t)\right\|}, p=\frac{e^{\prime}(t)}{\left\|e^{\prime}(t)\right\|}$ and $q=e(t) \wedge p(t)$ are the tangent, the principal normal and the binormal vector fields of the curve $\alpha$, respectively. The functions $\kappa$ and $\tau$ are called curvature and torsion of $\alpha$, respectively. The prime' denotes the differentiation with respect to the $t$-parameter.

The type 2 Bishop formulas of $\alpha$ are defined by

$$
\frac{\partial}{\partial t}\left[\begin{array}{c}
N_{1}(t) \\
N_{2}(t) \\
q(t)
\end{array}\right]=\left[\begin{array}{ccc}
0 & 0 & -\kappa_{1}(t) \\
0 & 0 & -\kappa_{2}(t) \\
\kappa_{1}(t) & \kappa_{2}(t) & 0
\end{array}\right]\left[\begin{array}{c}
N_{1}(t) \\
N_{2}(t) \\
q(t)
\end{array}\right] .
$$

For this frame, the vectors $N_{1}, N_{2}$ and $q$ are the tangent, the principal normal, and the binormal vector fields of the curve $\alpha$.

Here, the type 2 Bishop curvatures are defined by

$$
\begin{aligned}
& \kappa_{1}(t)=-\tau \cos \theta(t), \\
& \kappa_{2}(t)=-\tau \sin \theta(t) .
\end{aligned}
$$

It can be also shown that

$$
\theta^{\prime}=\kappa=\frac{f^{\prime}}{1+(f)^{2}}, \quad f=\frac{\kappa_{2}}{\kappa_{1}} .
$$

We shall call the set $\left\{N_{1}, N_{2}, q, \kappa_{1}, \kappa_{2}\right\}$ as type 2 Bishop invariants of the curve $\alpha=$ $\alpha(t)$.

The Bishop frame or parallel transport frame is an alternative to the Frenet frame. Thus, the matrix relation between type 2 Bishop and Frenet-Serret frames can be expressed as

$$
\left[\begin{array}{l}
e(t) \\
p(t) \\
q(t)
\end{array}\right]=\left[\begin{array}{ccc}
\sin \theta(t) & -\cos \theta(t) & 0 \\
\cos \theta(t) & \sin \theta(t) & 0 \\
0 & 0 & 1
\end{array}\right]\left[\begin{array}{c}
N_{1}(t) \\
N_{2}(t) \\
q(t)
\end{array}\right]
$$

We denote a surface $M$ in $\mathbf{E}^{3}$ by

$$
\phi=\phi(s, t) .
$$

Let $\zeta$ be the standard unit normal vector field on the surface $M$ defined by

$$
\zeta=\frac{\phi_{s} \wedge \phi_{t}}{\left\|\phi_{s} \wedge \phi_{t}\right\|}, \quad \phi_{s}=\frac{\partial \phi}{\partial s}, \quad \phi_{t}=\frac{\partial \phi}{\partial t} .
$$


Thus, we have the metric $g_{i j}$ and the coefficients of the second fundamental form $h_{i j}, i, j=1,2$

$$
\begin{aligned}
& g_{11}=\left\langle\phi_{s}, \phi_{s}\right\rangle, g_{12}=\left\langle\phi_{s}, \phi_{t}\right\rangle, g_{22}=\left\langle\phi_{t}, \phi_{t}\right\rangle . \\
& h_{11}=\left\langle\phi_{s s}, \zeta\right\rangle, h_{12}=\left\langle\phi_{s t}, \zeta\right\rangle, h_{22}=\left\langle\phi_{t t}, \zeta\right\rangle,
\end{aligned}
$$

where $\langle$,$\rangle is the Euclidean inner product.$

Under this parametrization of the surface $M$, the Gaussian curvature $K$ and the mean curvature $H$ have the following forms [18]

$$
\begin{gathered}
K=\frac{\operatorname{Det}\left(h_{i j}\right)}{\operatorname{Det}\left(g_{i j}\right)}, \\
H=\frac{1}{2} \operatorname{tr}\left(g^{i j} h_{j k}\right),
\end{gathered}
$$

where $\left(g^{k l}\right)$ is the associated contravariant metric tensor field of the covariant metric tensor field $\left(g_{k l}\right)$; that is, $g^{i k} g_{j k}=\delta_{j}^{i}$.

A surface $M$ in a three-dimensional Euclidean space $\mathbf{E}^{3}$ with positive Gaussian curvature $K$ possesses a positive definite second fundamental form $I I$ if appropriately orientated. Therefore, the second fundamental form defines a new Riemannian metric on $M$. In turn, we can consider the Gaussian curvature $K_{I I}$ of the second fundamental form which is regarded as a Riemannian metric. If a surface has non-zero Gaussian curvature everywhere, $K_{I I}$ can be defined formally and it is the curvature of the Riemannian manifold $(M, I I)$.

Definition 2.1. Given a surface $M$ in the three- dimensional Euclidean space $\mathbf{E}^{3}$, the second Gaussian curvature is defined by [3]

$$
\begin{aligned}
& K_{I I}=\frac{1}{\left(h_{11} h_{22}-h_{12}^{2}\right)^{2}}
\end{aligned}
$$

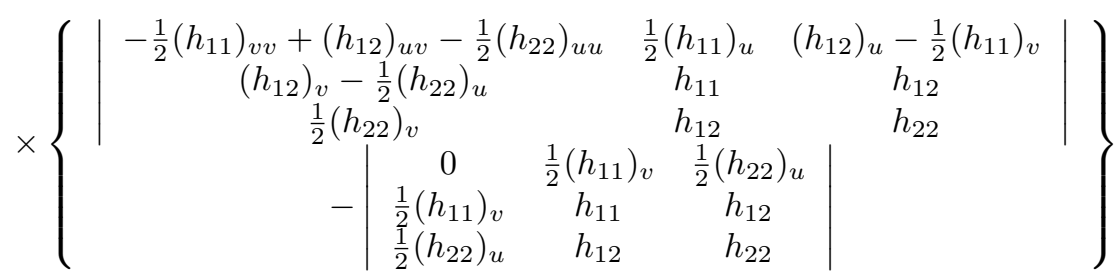

Now, to serve our study it is important to consider the following definition:

Definition 2.2. [30] (1) A regular surface is flat (developable) if and only if its Gaussian curvature is identically zero.

(2) A regular surface for which the mean curvature vanishes identically is minimal surface.

(3) A non -developable surface is said to be $I I$-flat if the second Gaussian curvature is equal to zero.

(4) A non- developable surface is called II -minimal if the second mean curvature is vanished. 


\section{Tube-like surface with type 2 Bishop frame in $\mathbf{E}^{3}$}

In this section, we study a special case of surfaces in 3 dimensions, i.e., a tubelike surface that is generated by sweeping a space curve along another central space curve.

The tube-like surface can be obtained from the tube surface which is a special kind of the canal surface.

A canal surface is the envelope of a moving sphere with varying radius defined by the trajectory $\alpha(t)$ (center curve) of its center and a radius function $r(t)$. If the radius function $r(t)$ is a constant, then the canal surface is called a tube [9].

For a sufficiently small parameter $r>0$ and by $\alpha(t)$ as a center curve with nonzero curvature, the tube-like surface of radius $r$ with type 2 Bishop formulas (2.3) can be written as

$$
\phi(s, t)=\alpha(t)+r\left[\cos s N_{2}(t)-\sin s q(t)\right],
$$

where in general $r$ can be a function of $t$. For fixed $t$, when $s$ runs from 0 to $2 \pi$, we have a circle around the point $\alpha(t)$ in the $N_{2} B$ plane. As we change $t$, this circle moves along the space curve $\alpha$, and we will generate a tube-like surface along $\alpha$.

Then, the two tangent vectors and the unit normal vector to the surface are given by

$$
\left\{\begin{array}{l}
\phi_{s}=-r\left[\sin s N_{2}+\cos s q\right] \\
\phi_{t}=\Omega N_{1}-r \kappa_{2}\left[\sin s N_{2}+\cos s q\right] \\
\zeta=-\cos s N_{2}+\sin s q, \quad \Omega=1-r \kappa_{1} \sin s
\end{array}\right.
$$

respectively. From (2.2) and (2.7) it is easily checked that the coefficients of the first fundamental form $g_{11}, g_{12}$ and $g_{22}$ of $\phi$ are given by

$$
g_{11}=r^{2}, \quad g_{12}=r^{2} \kappa_{2}, \quad g_{22}=\Omega^{2}+r^{2} \kappa_{2}^{2},
$$

From this, we have

$$
g=r^{2}\left(\Omega^{2}+r^{2} \kappa_{2}^{2}\right)-\left(r^{2} \kappa_{2}\right)^{2} .
$$

This leads to the coefficients of the second fundamental form $h_{11}, h_{12}$ and $h_{22}$ of $\phi$ given by

$$
h_{11}=r, h_{12}=r \kappa_{2}, h_{22}=r \kappa_{2}^{2}-\kappa_{1} \sin s+r \kappa_{1}^{2} \sin ^{2} s .
$$

It follows that

$$
h=r\left(r \kappa_{2}^{2}-\kappa_{1} \sin s+r \kappa_{1}^{2} \sin ^{2} s\right)-\left(r \kappa_{2}\right)^{2} .
$$

Besides, the Gaussian curvature $K$ and the mean curvature $H$ of (3.1) are respectively, given by

$$
\begin{gathered}
K=-\frac{\kappa_{1} \cos s}{\Omega r}, \\
H=\frac{1-2 r \kappa_{1} \cos s}{2 \Omega r} .
\end{gathered}
$$

If the second fundamental form of $\phi$ is non-degenerate, i.e., $h_{11} h_{22}-\left(h_{12}\right)^{2} \neq 0$, then the second Gaussian curvature $K_{I I}$ on $\phi(s, t)$ can be obtained

$$
K_{I I}=\frac{1}{4 \Omega^{4} r \sin ^{2} s}\left[1+\sin ^{2} s-6 r \kappa_{1} \sin ^{3} s+4 r^{2} \kappa_{1}^{2} \sin ^{4} s\right] .
$$




\subsection{Tube-like surface of W- type}

In the following, we study the tube-like surface $\phi$ in $\mathbf{E}^{3}$ satisfying the Jacobi equation $\Gamma(X, Y)=0, X \neq Y$, of the curvatures $K, H$ and $K_{I I}$ of $\phi$ and we formulate the main results in the next theorems.

Theorem 3.1. Let $M$ be a tube-like surface in $\mathbf{E}^{3}$ defined by Eq. (3.1), then $M$ is a $(K, H) W$-surface.

Proof. Let $M$ be a tube-like surface in $\mathbf{E}^{3}$. Differentiating $K$ and $H$ with respect to $s$ and $t$ respectively, then we obtain

$$
\begin{aligned}
& K_{s}=-\frac{\kappa_{1} \cos s}{r \Omega^{2}}, \quad K_{t}=-\frac{\kappa_{1}^{\prime} \sin s}{r \Omega^{2}}, \\
& H_{s}=-\frac{\kappa_{1} \cos s}{2 \Omega^{2}}, \quad H_{t}=-\frac{\kappa_{1}^{\prime} \sin s}{2 \Omega^{2}} .
\end{aligned}
$$

By using (3.8) and (3.9), $M$ satisfies identically the Jacobi equation

$$
\phi(K, H)=K_{s} H_{t}-K_{t} H_{s}=0 .
$$

Therefore $M$ is a $W$-surface.

Theorem 3.2. Let $M$ be a tube-like surface parameterized by (3.1) with non-degenerate second fundamental form in the Euclidean 3-space $E^{3}$. If $M$ is a $\left(K, K_{I I}\right) W$-surface, then $\kappa_{1}^{\prime}=0$, i.e., the curvature of $\alpha(t)$ is a non-zero constant.

Proof. Let $M$ be a tube-like surface in $\mathbf{E}^{3}$ parameterized by (3.1). If we take derivative of $K_{I I}$ given by (3.7) with respect to $s$ and $t$ respectively, and using Eq. (3.8) then we have

$$
\begin{gathered}
\left(K_{I I}\right)_{s}=\frac{-1}{2 r \Omega^{3} \sin ^{3} s}\left[1-r \kappa_{1}\left(2 \cos ^{2} s+r \kappa_{1} \sin ^{3} s\right) \sin s\right] \cos s, \\
\left(K_{I I}\right)_{t}=\frac{\kappa_{1}^{\prime}}{2 \Omega^{3} \sin s}\left[\cos ^{2} s-\sin ^{2} s+r \kappa_{1} \sin ^{3} s\right] .
\end{gathered}
$$

We consider the tube-like surface (3.1) in $\mathbf{E}^{3}$ satisfying the Jacobi equation

$$
\phi\left(K, K_{I I}\right)=K_{s}\left(K_{I I}\right)_{t}-K_{t}\left(K_{I I}\right)_{s}=0,
$$

with respect to the Gaussian curvature $K$ and the second Gaussian curvature $K_{I I}$. Then, substituting from (3.10) and (3.11) into (3.12), we get

$$
\kappa_{1}^{\prime} \cos s=0 .
$$

Since this polynomial is equal to zero for every $s$, its coefficient must be zero. Therefore, we conclude that $\kappa_{1}^{\prime}=0$.

Theorem 3.3. Let $M$ be a tube-like surface parameterized by (3.1) with non-degenerate second fundamental form in the Euclidean 3-space $\mathbf{E}^{3}$. If $M$ is a $\left(H, K_{I I}\right) W$-surface, then $\kappa_{1}^{\prime}=0$. This means that the curvature of $\alpha(t)$ is a non-zero constant. 
Proof. We assume that the tube-like surface given by (3.1) with non-degenerate second fundamental form in $\mathbf{E}^{3}$ is a $\left(H, K_{I I}\right) W$-surface. Then, it satisfies the Jacobi equation

$$
\phi\left(H, K_{I I}\right)=H_{s}\left(K_{I I}\right)_{t}-H_{t}\left(K_{I I}\right)_{s}=0 .
$$

Equations (3.9), (3.10), (3.11) and (3.13) lead to

$$
\kappa_{1}^{\prime} \cos s=0 .
$$

From (3.14), one can get $\kappa_{1}^{\prime}=0$. Thus, the curvature of $\alpha(t)$ is a non-zero constant.

\section{Tube-like surface of LW- type}

Now, to examine the linear Weingarten property of the tube-like surface $\phi$ defined along the space curve $\alpha(t)$. Let us analyze the following theorems.

Theorem 4.1. Suppose that the tube-like surface defined by (3.1) in $\mathbf{E}^{3}$ is a $L W$-surface satisfying $a K+b H=c$, then $\kappa_{1}=0$ and $M$ is an open part of a circular-like cylinder.

Proof. Consider the parametrization (3.1) with $K$ and $H$ given by (3.5) and (3.6) respectively, then the relation

$$
a K+b H=c,
$$

implies

$$
2 \kappa_{1}\left(a+b r-c r^{2}\right) \sin s-b+2 c r=0 .
$$

Since $\sin s$ and 1 are linearly independent, we have

$$
2 \kappa_{1}\left(a+b r-c r^{2}\right)=0, \quad b=2 c r .
$$

This leads to

$$
\kappa_{1}\left(a+c r^{2}\right)=0 .
$$

If $a+c r^{2} \neq 0$, then $\kappa_{1}=0$. Thus, $M$ is an open part of a circular-like cylinder.

Theorem 4.2. Let $(A, Q) \in\left\{\left(K, K_{I I}\right),\left(H, K_{I I}\right)\right\}$, then there are no $(A, Q) L W$-tubelike surfaces in Euclidean 3-space $\mathbf{E}^{3}$.

Proof. Firstly, we suppose that the tube-like surface (3.1) with non-degenerate second fundamental form in $\mathbf{E}^{3}$. satisfies the equation

$$
a K+b K_{I I}=c .
$$

By the aid of (3.5) and (3.7), the equation (4.2) takes the form

$$
\begin{gathered}
\frac{-1}{4 r \Omega^{2} \sin ^{2} s}\left[-4 r \kappa_{1}^{2}\left(a+b r-c r^{2}\right) \sin ^{4} s\right. \\
\left.+2 \kappa_{1}\left(2 a+3 b r-4 c r^{2}\right) \sin ^{3} s-(b-4 c r) \sin ^{2} s-b\right]=0 .
\end{gathered}
$$

Since the identity holds for every $s$, all the coefficients must be zero. Therefore, we obtain

$$
\begin{gathered}
4 r \kappa_{1}^{2}\left(a+b r-c r^{2}\right)=0, \\
2 \kappa_{1}\left(2 a+3 b r-4 c r^{2}\right)=0, \\
(b-4 c r)=0, \quad b=0 .
\end{gathered}
$$


Thus, we get $b=0, c=0$ and $\kappa_{1}=0$. In this case, the second fundamental form of $M$ is degenerate.

Secondly, let the tube-like surface (3.1) with non-degenerate second fundamental form in $\mathbf{E}^{3}$ satisfy the relation

$$
a H+b K_{I I}=c .
$$

From Equations (3.6), (3.7) and (4.3), we get

$$
\begin{gathered}
\frac{-1}{4 r \Omega^{2} \sin ^{2} s}\left[-4 r^{2} \kappa_{1}^{2}(a+b-c r) \sin ^{4} s\right. \\
+2 r \kappa_{1}(3 a+3 b-4 c r) \sin ^{3} s \\
\left.-(2 a+b-4 c r) \sin ^{2} s-b\right]=0 .
\end{gathered}
$$

Based on the above, one can obtain $b=0, c=0$ and $\kappa_{1}=0$. It indicates that the second fundamental form of the tube-like surface is degenerate. Then, there are no $\left(H, K_{I I}\right)$-linear Weingarten tube-like surfaces in $\mathbf{E}^{3}$.

\section{Application}

Now, as an application of our main results, we give the following example

Example 5.1. Consider the surface given by

$$
\phi(s, t)=\alpha(t)+r\left(\cos s N_{2}(t)-\sin s q(t)\right),
$$

where $\alpha(t)$ is given by

$$
\alpha(t)=(\cos t, \sin t, t) .
$$

The Bishop frame $\left\{N_{1}(t), N_{2}(t), q(t)\right\}$ of the curve $\alpha$ is expressed as

$$
\left\{\begin{array}{c}
N_{1}(t)=\frac{1}{\sqrt{2}}(-\sin t, \cos t, 1) \\
N_{2}(t)=-(\cos t, \sin t, 0) \\
q(t)=\frac{1}{\sqrt{2}}(\sin t,-\cos t, 1)
\end{array}\right.
$$

Thus, the parametric form of the tube-like surface $\phi(s, t)$ can be written as

$$
\phi(s, t)=\left(\lambda_{1}(s, t), \lambda_{2}(s, t), \lambda_{3}(s, t)\right)
$$

where

$$
\left\{\begin{array}{l}
\lambda_{1}=\left((1-r \cos s) \cos t-\frac{1}{\sqrt{2}} r \sin s \sin t\right), \\
\lambda_{2}=\left((1-r \cos s) \sin t+\frac{1}{\sqrt{2}} r \sin s \cos t\right), \\
\lambda_{3}=\left(t-\frac{1}{\sqrt{2}} r \sin s\right)
\end{array}\right.
$$

For this parametrization surface, the components of the first fundamental form are given by

$$
\left\{\begin{array}{l}
g_{11}=r^{2}, \quad g_{12}=\frac{-1}{2} r^{2} \sin \theta(t), \\
g_{22}=\frac{1}{4}\left[(2+r \sin s \cos \theta(t))^{2}+r^{2} \sin ^{2} \theta(t)\right] .
\end{array}\right.
$$

The unit normal vector of $\phi$ is obtained from (2.6) as

$$
\zeta=\cos s N_{2}(t)-\sin s q(t) .
$$


Then the second fundamental form components of $\phi$ are as follows:

$$
\left\{\begin{array}{l}
h_{11}=-r, \quad h_{12}=\frac{1}{2} r \sin \theta(t), \\
h_{22}=\frac{1}{4}\left(-2 \sin s \cos \theta(t)-r\left(\sin ^{2} s+\cos ^{2} \sin ^{2} \theta(t)\right) .\right.
\end{array}\right.
$$

In addition, the Gaussian curvature $K$ and the mean curvature $H$ of $\phi$ are respectively, given by

$$
\begin{gathered}
K=\frac{\sin \mathrm{s} \cos \theta(t)}{2 r+r^{2} \sin \mathrm{s} \cos \theta(t)} \\
H=\frac{-1}{r}+\frac{1}{r(2+r \sin s \cos \theta(t))} .
\end{gathered}
$$

Since $h_{11} h_{22}-h_{12}^{2} \neq 0$, then we can get the second Gaussian curvature $K_{I I}$ on $\phi(s, t)$ as follows:

$$
K_{I I}=-\frac{\cos ^{2} \mathrm{~s}+2 \sin ^{2} \mathrm{~s}+3 r \sin ^{3} \mathrm{~s} \cos \theta(t)+r^{2} \sin ^{4} \mathrm{~s} \cos \theta(t)}{r(2+r \sin \mathrm{s} \cos \theta(t))^{2} \sin ^{2} \mathrm{~s}} .
$$

From aforementiomed data, one can deduce that when $\sin s=0$, then from (5.9) and (5.10), we get $K=0$ and $H=\frac{-1}{2 r}=$ const., respectively.

Therefore, in the three dimensional Euclidean space $\mathbf{E}^{3}$, equations (5.9)-(5.11) show that:

The surface (5.4) is a $(K, H) W$-surface (Theorem 3.1.).

Besides, it is $\left(K, K_{I I}\right)$ and $\left(H, K_{I I}\right) W$-surface (Theorems 3.2 and 3.3).

Moreover, it is an open part of a circular-like cylinder (Theorem 4.1).

In addition, there are no tube-like surfaces of types $\left(K, K_{I I}\right)$ and $\left(H, K_{I I}\right)$ $L W$-surface (Theorem 4.2).

We can easily see the graph of some tube-like surfaces generated by circular helix in Figures 1, 2, 3.

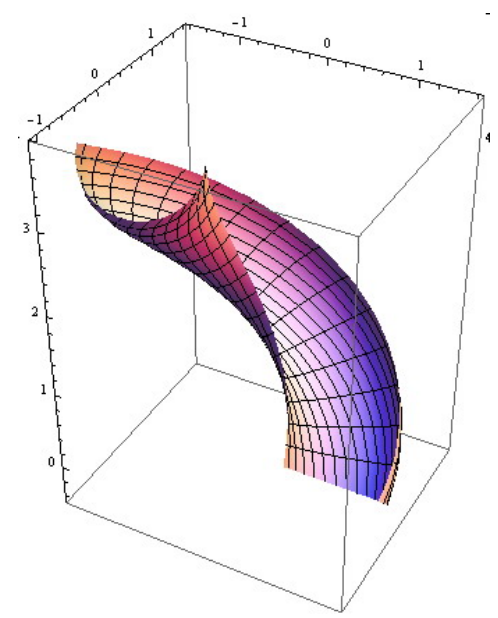

Figure 1. Tube-like surface generated by circular helix with $s \in[0,1.1 \pi], t \in[0,1.2 \pi]$

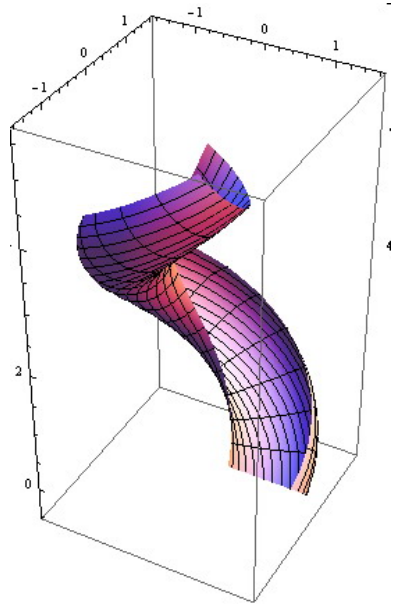

Figure 2. Tube-like surface generated by circular helix with $s \in[0,1.2 \pi], t \in[0,1.7 \pi]$

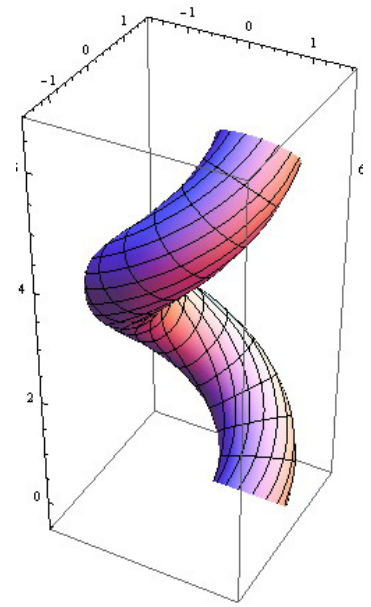

Figure 3. Tube-like surface generated by circular helix with $s \in[0,2 \pi], t \in[0,2 \pi]$ 


\section{Conclusion}

In this paper, we proposed a definition of a tube-like surface in the threedimensional Euclidean space $E^{3}$. It is generated by sweeping a space curve along another central space curve. We investigated the meant surface on satisfying some equations in terms of the Gaussian curvature $K$, the mean curvature $H$ and the second Gaussian curvatures $K_{I I}$ using a new version of Bishop frame. As an application to demonstrate our theoretical results, we have given an example.

\section{References}

[1] Abdel-Aziz, H.S., Khalifa Saad, M., Weingarten timelike tube surfaces around a spacelike curve, Int. J. of Math. Analysis, 25(2011), no. 5, 1225-1236.

[2] Abdel-Aziz, H.S., Khalifa Saad, M., Sezai Kiziltug, Parallel Surfaces of Weingarten Type in Minkowski 3-Space, Int. Math. Forum, 4(2012), no. 7, 2293-2302.

[3] Baikoussis, C., Koufogiorgos, T., On the inner curvature of the second fundamental form of helicoidal surfaces, Arch. Math., 68(1997), no. 2, 169-176.

[4] Bishop, L.R., There is more than one way to frame a curve, The American Mathematical Monthly, 82(1975), no. 3, 246-251.

[5] Bükcü, B., Karacan, M.K., Special Bishop motion and Bishop Darboux rotation axis of the space curve, The Journal of Dynamical Systems and Geometric Theories, 6(2008), 27-34.

[6] Bükcü, B., Karacan, M.K., On the slant helices according to Bishop frame of the timelike curve in Lorentzian space, Tamkang Journal of Mathematics, 39(2008), no. 3, 255-262.

[7] Bükcü, B., Karacan, M.K., The slant helices according to Bishop frame, International Journal of Computational and Mathematical Sciences, 3(2009), no. 2, 67-70.

[8] Dillen, F., Kühnel, W., Ruled Weingarten surfaces in a Minkowski 3-space, Manuscripta Math, 98(1999), 307-320.

[9] Doğan, F., A note on tubes, Int. J. of Phys. and Math. Sciences, 3(2013), no. 1, 98-105.

[10] Karacan, M.K., Bükcü, B., Yuksel, N., On the dual Bishop Darboux rotation axis of the dual space curve, Applied Sciences, 10(2008), 115-120.

[11] Kiziltuğ, S., On characterization of inextensible flows of curves according to type-2 Bishop frame $E^{3}$, Mathematical and Computational Applications, 19(2014), no. 1, 69-77.

[12] Kim, H.Y., Yoon, D.W., Classification of ruled surfaces in a Minkowski 3-space, J. Geom. Phys., 49(2004), 89-100.

[13] Kim, H.Y., Yoon, D.W., Weingarten quadric surfaces in a Euclidean 3-space, Turk. J. Math, 35 (2011), 479-485.

[14] Lopez, R., On linear Weingarten surfaces, International J. Math., 19(2008), 439-448.

[15] Lopez, R., Special Weingarten surfaces foliated by circles, Monatsh. Math., 154(2008), 289-302.

[16] Lopez, R., Parabolic Weingarten surfaces in hyperbolic space, Publ. Math. Debrecen, 74(2009), 59-80.

[17] Munteanu, M.I., Nistor, A.I., Polynomial translation Weingarten surfaces in 3dimensional Euclidean space, Differential geometry, Worled Sci. Publ., Hackensack, NJ, (2009), 316-320. 
[18] O'Neill, B., Elementary Differential Geometry, Academic Press, New York, 1966.

[19] Ozyilmaz, E., Classical differential geometry of curves according to type-2 Bishop trihedra, Mathematical \& Computational Applications, 16(2011), no. 4, 858-867.

[20] Petrović, M., Verstraelen, J., Verstraelen, L., Principal normal spectral variations of space curves, Proyecciones, 19(2000), no. 2, 141-155.

[21] Ro, J., Yoon, D.W., Tubes of Weingarten types in a Euclidean 3-space, J. Chungcheong Math. Soc., 22(2009), no. 3, 359-366.

[22] Shifrin, T., Differential geometry: A first course in curves and surfaces, Preliminary Version, Springer, 2015.

[23] Souror, A.H., Weingarten tube-like surfaces in Euclidean 3-space, Stud. Univ. BabeşBolyai Math., 61(2016), no. 2, 239-250.

[24] Sodsiri, W., Ruled surfaces of Weingarten type in Minkowski 3-space, PhD. thesis, Katholieke Universiteit Leuven, Belgium, 2005.

[25] Süha, Y., Bishop spherical images of a spacelike curve in Minkowski 3-space, International Journal of Physical Sciences, 5(2010), no. 6, 898-905.

[26] Van-Brunt, Grant, K., Potential applications of Weingarten surfaces in CAGD, Part I: Weingarten surfaces and surface shape investigation, Comput. Aided Geom. Des, 13(1996), 569-582.

[27] Weingarten, J., Ueber eine Klasse auf einander abwickelbarer Flaachen, J. Reine Angew. Math., 59(1861), 382-393.

[28] Weingarten, J., Ueber die Flachen derer Normalen eine gegebene Flache beruhren, J. Reine Angew. Math., 62(1863), 61-63.

[29] Yılmaz, S., Turgut, M., A new version of Bishop frame and an application to spherical images, Journal of Mathematical Analysis and Applications, 371(2010), no. 2, 764-776.

[30] Yilmaz, T., Murat, K.K., Differential Geometry of three dimensions, Syndic of Cambridge University Press, 1981.

[31] Yoon, D.W., Jun, J.S., Non-degenerate quadric surfaces in Euclidean 3-space, Int. J. Math. Anal., 6(2012), no. 52, 2555-2562.

Hossam S. Abdel-Aziz

Department of Mathematics

Faculty of Science

Sohag University

82524, Sohag, Egypt

e-mail: habdelaziz2005@yahoo.com 